\title{
GAUSS MAPS OF TRANSLATING SOLITONS OF MEAN CURVATURE FLOW
}

\author{
CHAO BAO AND YUGUANG SHI
}

(Communicated by Lei $\mathrm{Ni}$ )

\begin{abstract}
In this short note we study the Bernstein type theorem of translating solitons whose images of their Gauss maps are contained in compact subsets in an open hemisphere of the standard $\mathbf{S}^{n}$. As a special case we get a classical Bernstein type theorem in minimal submanifolds in $\mathbf{R}^{n+1}$.
\end{abstract}

\section{INTRODUCTION}

Let $F_{0}: \boldsymbol{\Sigma} \rightarrow \mathbf{R}^{n+1}$ be a smooth immersion of an n-dimensional hypersurface in $\mathbf{R}^{n+1}, n \geq 2$. The mean curvature flow is a one-parameter family of smooth immersions $F: \boldsymbol{\Sigma} \times[0, T) \rightarrow \mathbf{R}^{n+1}$ satisfying:

$$
\left\{\begin{array}{l}
\frac{\partial F}{\partial t}(p, t)=-H(p, t) \vec{\nu}, p \in M, t \geq 0 \\
F(\cdot, 0)=F_{0}
\end{array}\right.
$$

where $-H(p, t) \vec{\nu}$ is the mean curvature vector, $\vec{\nu}(p, t)$ is the outer normal vector and $H(p, t)$ is the mean curvature with respect to the normal vector $\vec{\nu}(p, t)$. It is easy to see that the mean curvature of a convex surface is positive in our definition.

If the initial hypersurface is compact, it is not hard to see that mean curvature flow must develop singularities in finite time. By the blow up rate of second fundamental form, we can divide the singularities into two types: i.e. we say it is a type-1 singularity if there is a constant $C$ such that

$$
\max _{M_{t}}|A|^{2} \leq \frac{C}{T-t} \text { as } t \rightarrow T
$$

otherwise we say it is of type 2. Here $A$ is the second fundamental form of hypersurface at time $t$. It is well-known that if a mean curvature flow develops type- 1 singularities, we can get self-shrinking solutions after rescaling the surface near a singularity. Similarly, if the initial surface is mean convex and the singularity is of type 2, Huisken and Sinistrari [5] have proved that any limiting flow is a convex hypersurface which is a convex translating soliton. Also, it is not too difficult to see that a translating soliton is a hypersurface in $\mathbf{R}^{n+1}$ satisfying certain nonlinear equations (see (2) below, and we only consider the codimension 1 case in this paper). It plays an important role in analysis of singularities in mean curvature

Received by the editors January 21, 2013.

2010 Mathematics Subject Classification. Primary 53C44; Secondary 58J05.

Key words and phrases. Translating soliton, Gauss map.

This research was partially supported by NSF of China grant No. 10990013. 
flow (see [4,5] for example). On the other hand, translating solitons can also be regarded as natural generalizations of a minimal hypersurface in $\mathbf{R}^{n+1}$. With these facts in mind, it is natural to ask if Bernstein's type result is still true for translating solitons.

Inspired by [1, 7, 9 11, we focus on the investigation of uniqueness translating soliton solutions through their Gauss maps. We are able to get a Bernstein type property of translating solitons. Namely, if the Gauss map image of a translating soliton lies in a compact subset of an open hemisphere of $\mathbf{S}^{n}$, then it must be a hyperplane. For self-shrinkers similar results have been obtained by Xin, Ding, and Yang [10] (Theorem 3.2).

Before we state our main results, we need to recall some basic facts and notation.

A translating soliton is a solution to (11) translating in the direction of a constant vector $T$ in $\mathbf{R}^{n+1}$; more precisely, we say $F: \boldsymbol{\Sigma}^{n} \rightarrow \mathbf{R}^{n+1}$ is a translating soliton if $\langle T, \vec{\nu}(p)\rangle=-H(p)$ (here $\vec{\nu}(p)$ and $H(p)$ are defined as above). For simplicity, we will identify $F(\boldsymbol{\Sigma})$ and $\boldsymbol{\Sigma}$ in the sequel and simply say $\boldsymbol{\Sigma}$ is a translating soliton. For a vector $X=X^{\alpha} \frac{\partial}{\partial z^{\alpha}}$ in $\mathbf{R}^{n+1}$, it is simply denoted by $\left\{X^{\alpha}\right\}$ in the sequel, where $\frac{\partial}{\partial z^{\alpha}}$ is the standard Cartesian coordinate of $\mathbf{R}^{n+1}$.

Let $\boldsymbol{\Sigma}$ be a translating soliton. We always take the outer normal vector throughout this paper, denote the induced metric by $g=\left\{g_{i j}\right\}$, the surface measure by $d \mu$, the second fundamental form by $A=\left\{h_{i j}\right\}$, and the mean curvature by $H=g^{i j} h_{i j}$. We then denote by $\lambda_{1} \leq \cdots \leq \lambda_{n}$ the principal curvature, i.e. the eigenvalue of the matrix $\left(h_{j}^{i}\right)=\left(g^{i k} h_{k j}\right)$. It is obvious that $H=\lambda_{1}+\cdots+\lambda_{n}$. In addition, $|A|^{2}=\lambda_{1}^{2}+\cdots+\lambda_{n}^{2}$ will denote the squared norm of the second fundamental form.

Let $u: \Sigma \rightarrow \mathbf{S}^{n}$ be the Gauss map of the translating soliton $\boldsymbol{\Sigma}$, and the image $u(x)$ be the unit outer normal vector of $\boldsymbol{\Sigma}$.

Theorem 1.1. Let $\boldsymbol{\Sigma} \subset \mathbf{R}^{n+1}$ be an $n$-dimensional complete translating soliton with bounded mean curvature. If the image of a Gauss map $u$ of $\boldsymbol{\Sigma}$ lies in a ball $B_{\Lambda}^{S^{n}}\left(y_{0}\right)$ of $\mathbf{S}^{n}$, where $\Lambda<\frac{\pi}{2}$, then $\boldsymbol{\Sigma}$ must be a hyperplane. Here and in the sequel, $B_{\lambda}^{S^{n}}\left(y_{0}\right)$ denotes the geodesic ball of radius $\Lambda$ and with center $y_{0}$ in $\mathbf{S}^{n}$.

As mentioned earlier, a complete minimal hypersurface is a stationary solution of mean curvature flow. Because it is also a translating soliton, we can also get a Bernstein type result for minimal hypersurfaces like the following one. Indeed, it is a classical result due to J. Moser [8].

Corollary 1.2. Let $\boldsymbol{\Sigma} \subset \mathbf{R}^{n+1}$ be a complete minimal hypersurface and let the image of Gauss map $u$ of $\boldsymbol{\Sigma}$ lie in a ball $B_{\Lambda}^{S^{n}}\left(y_{0}\right)$ of $\mathbf{S}^{n}$, where $\Lambda<\frac{\pi}{2}$. Then $\boldsymbol{\Sigma}$ must be a hyperplane.

Remark 1.3. By assuming $\boldsymbol{\Sigma}$ has Euclidean volume growth and the image under the Gauss map omits a neighbourhood of $\overline{\mathbf{S}}_{+}^{n-1}$, Jost, Xin, and Yang could prove a similar result (see Theorem 6.6 in [3]). We suspect that our assumption on the image of a Gauss map implies Euclidean volume growth of $\boldsymbol{\Sigma}$.

In the remainder of the paper we will first derive some useful formulae for translating solitons and then give a proof of Theorem 1.1 


\section{The Gauss map of a translating soliton}

Let $V=\left\{V^{\alpha}\right\}$ be the tangential part of $T$; then its normal component must be $-H \nu^{\alpha}$ where $\left\{\nu^{\alpha}\right\}$ denotes the unit outer normal vector field. In local coordinates

$$
V^{\alpha}=V^{i} \nabla_{i} F^{\alpha}
$$

Take the covariant derivative on the above equality for $i=1, \cdots, n$ :

$$
\begin{aligned}
0 & =\nabla_{i} T^{\alpha}=\nabla_{i}\left(V^{j} \frac{\partial F^{\alpha}}{\partial x_{j}}-H N^{\alpha}\right) \\
& =\left(\nabla_{i} V^{j}\right) \frac{\partial F^{\alpha}}{\partial x_{j}}+V^{j}\left(-h_{i j} N^{\alpha}\right)-\left(\nabla_{i} H\right) N^{\alpha}-H h_{i j} g^{j k} \frac{\partial F^{\alpha}}{\partial x_{k}}
\end{aligned}
$$

Then equating tangential and normal components we find that

$$
\left\{\begin{array}{l}
\nabla_{i} V^{j}=H h_{i k} g^{k j} \\
\nabla_{i} H+h_{i j} V^{j}=0
\end{array}\right.
$$

We use the previous definition of Gauss map $u: \boldsymbol{\Sigma} \rightarrow \mathbf{S}^{n}$. The pullback under $u$ of the tangent bundle $T \mathbf{S}^{n}$ to a bundle over $u$ is denoted by $u^{-1} T \mathbf{S}^{n} . T \boldsymbol{\Sigma}$ and $N \boldsymbol{\Sigma}$ denote the tangent bundle and the normal bundle of $\boldsymbol{\Sigma}$, respectively. It is easy to see that $u^{-1} T \mathbf{S}^{n}$ is isometric to the tensor product $T \boldsymbol{\Sigma} \times N \boldsymbol{\Sigma}$.

By definition, the mean curvature $H=T r u_{*}$ is the trace of $u_{*}$ with respect to the Riemannian metric on $T \boldsymbol{\Sigma}$. We denote $d u=u_{*}$, and obviously $d u$ is a cross section on $u^{-1} T \mathbf{S}^{n}$.

So we can get that $\nabla H=\nabla \operatorname{Tr} d u=\operatorname{Tr} \nabla d u$. Now, let us compute exactly what $\operatorname{Tr} \nabla d u$ is. Denote by $\Gamma$ and $\tilde{\Gamma}$ the Christoffel symbols on $\boldsymbol{\Sigma}$ and $\mathbf{S}^{n}$, and by $\left\{x^{i}\right\}$ and $\left\{y^{\alpha}\right\}$ the local coordinates on $\boldsymbol{\Sigma}$ and $\mathbf{S}^{n}$ respectively:

$$
\begin{aligned}
\nabla d u & =\nabla_{j}(d u) d x^{j}=\nabla_{j}\left(\frac{\partial u^{\alpha}}{\partial x^{i}} d x^{i} \otimes \frac{\partial}{\partial y^{\alpha}}\right) d x^{j} \\
& =\left(\frac{\partial^{2} u^{\alpha}}{\partial x^{i} \partial x^{j}} d x^{i} \otimes \frac{\partial}{\partial y^{\alpha}}-\Gamma_{l j}^{i} \frac{\partial u^{\alpha}}{\partial x^{i}} d x^{l} \otimes \frac{\partial}{\partial y^{\alpha}}+\tilde{\Gamma}_{\alpha \beta}^{\sigma} \frac{\partial u^{\alpha}}{\partial x^{i}} \frac{\partial u^{\beta}}{\partial x^{j}} d x^{i} \otimes \frac{\partial}{\partial y^{\sigma}}\right) d x^{j}
\end{aligned}
$$

So,

$$
\operatorname{Tr} \nabla d u=\Delta u^{\alpha} \frac{\partial}{\partial y^{\alpha}}+g^{i j} \tilde{\Gamma}_{\beta \sigma}^{\alpha} \frac{\partial u^{\beta}}{\partial x^{i}} \frac{\partial u^{\sigma}}{\partial x^{j}} \frac{\partial}{\partial y^{\alpha}} .
$$

We denote

$$
\tau^{\alpha}(u)=\Delta u^{\alpha}+g^{i j} \tilde{\Gamma}_{\beta \sigma}^{\alpha} \frac{\partial u^{\beta}}{\partial x^{i}} \frac{\partial u^{\sigma}}{\partial x^{j}} .
$$

By the previous calculations, we get $\tau(u)=\nabla H$.

Choose an orthonormal frame $\left\{e_{i}\right\}_{i=1}^{n}$ on $\boldsymbol{\Sigma}$. From (2) and the Weingarten formula,

$$
\nabla_{i} u=h_{i j} e_{j},
$$

we get $\nabla H=-d u(V)$. Then we have the following lemma:

Lemma 2.1. The Gauss map u of a translating soliton $\boldsymbol{\Sigma}$ forms a quasi-harmonic map, i.e.

$$
\tau(u)=-d u(V),
$$

where $\tau(u)$ is defined as above, and $V$ is a tangent vector field on $\mathbf{\Sigma}$. 
Lemma 2.2. For the quasi-harmonic equation of a Gauss map u, we have the following Bochner formula:

$$
\Delta|\nabla u|^{2}=2|\nabla d u|^{2}-2|\nabla u|^{4}-\left\langle V, \nabla|\nabla u|^{2}\right\rangle .
$$

Proof. Denote $R_{i j k l}$ and $K_{\alpha \beta \gamma \sigma}$ as the curvature operators on $\boldsymbol{\Sigma}$ and $\mathbf{S}^{n}$, respectively, both with induced metric. In general, we have the Bochner formula (see Lemma 3.1 in [6]):

$$
\Delta|\nabla u|^{2}=2|\nabla d u|^{2}+2\langle d \tau(u), d u\rangle+2 R_{i j}\left\langle u_{i}, u_{j}\right\rangle-2 K_{\alpha \beta \gamma \sigma} u_{i}^{\alpha} u_{j}^{\beta} u_{i}^{\gamma} u_{j}^{\sigma} .
$$

By the Gauss equation for a hypersurface, we have

$$
\begin{gathered}
R_{i j}=H h_{i j}-h_{i k} h_{k j}, \\
K_{\alpha \beta \gamma \sigma}=\delta_{\alpha \gamma} \delta_{\beta \sigma}-\delta_{\alpha \sigma} \delta_{\beta \gamma} .
\end{gathered}
$$

Because $u$ is a Gauss map and an outer normal vector, the Weingarten formula gives $u_{i}=h_{i j} e_{j}$. Together with (2), we compute

$$
\begin{aligned}
\langle d \tau(u), d u\rangle & =\langle d u, d(-d u(V))\rangle=-\langle d u, d u(\nabla V)\rangle-\left\langle d u, \nabla_{V} d u\right\rangle \\
& =-H A(\nabla u, \nabla u)-\frac{1}{2}\left\langle V, \nabla|\nabla u|^{2}\right\rangle .
\end{aligned}
$$

Using the Gauss equation, we have

$$
\begin{aligned}
R_{i j}\left\langle u_{i}, u_{j}\right\rangle & =H A(\nabla u, \nabla u)-h_{i k} h_{k j} h_{i p} h_{j p} \\
& =H A(\nabla u, \nabla u)-\sum_{i, j}\left(\sum_{k} h_{i k} h_{k j}\right)^{2}
\end{aligned}
$$

and

$$
\begin{aligned}
K_{\alpha \beta \gamma \sigma} u_{i}^{\alpha} u_{j}^{\beta} u_{i}^{\gamma} u_{j}^{\sigma} & =\delta_{\alpha \gamma} \delta_{\beta \sigma} u_{i}^{\alpha} u_{j}^{\beta} u_{i}^{\gamma} u_{j}^{\sigma}-\delta_{\alpha \sigma} \delta_{\beta \gamma} u_{i}^{\alpha} u_{j}^{\beta} u_{i}^{\gamma} u_{j}^{\sigma} \\
& =|\nabla u|^{4}-\sum_{i, j}\left(\left\langle u_{i}, u_{j}\right\rangle\right)^{2} \\
& =|\nabla u|^{4}-\sum_{i, j}\left(\sum_{k} h_{i k} h_{k j}\right)^{2} .
\end{aligned}
$$

The assertion follows from the above equalities.

Lemma 2.3. On any geodesic ball $B_{\Lambda}^{S^{n}}\left(y_{0}\right)$ of $\mathbf{S}^{n}, \Lambda<\frac{\pi}{2}$, let $\rho$ be the distance function from $y_{0}$ on $\mathbf{S}^{n}$. We define $\varphi(y)=1-\cos \rho(y)$ on $B_{\Lambda}^{S^{n}}\left(y_{0}\right)$. Then $\varphi$ satisfies the following properties:

(1) There exists a constant $b$ such that $0 \leq \varphi<b<1$;

(2) $\frac{d \varphi}{d \rho}=\sin \rho$;

(3) Hess $\varphi=(\cos \rho) I$, where Hess $\varphi$ is the hessian of $\varphi$, and $I$ is the identity matrix.

Proof. (1) and (2) hold obviously, so we only need to prove (3). Applying Proposition 2.20 of [2] (or see 1]),

$$
D^{2} \rho=\frac{\cos \rho}{\sin \rho}\left(d S^{2}-d \rho \otimes d \rho\right),
$$

where $d S^{2}$ is the metric tensor on $\mathbf{S}^{n}$. It is easy to check that $D^{2} \varphi=\varphi^{\prime} D^{2} \rho+$ $\varphi^{\prime \prime} d \rho \otimes d \rho$; thus we get $D^{2} \varphi=(\cos \rho) d S^{2}$, i.e. $\varphi_{i j}=(\cos \rho) \delta_{i j}$. 
Proof of Theorem 1.1. Choosing a convex function on $B_{\Lambda}^{S^{n}}\left(y_{0}\right)$ as above, by direct computation we have

$$
\Delta \varphi(u(x))=\operatorname{Hess} \varphi(\nabla u, \nabla u)+\langle D \varphi, \tau(u)\rangle=\cos \rho|\nabla u|^{2}-\langle V, \nabla \varphi\rangle .
$$

Define $\phi(x)=\frac{|\nabla u|^{2}(x)}{(b-\varphi(u(x)))^{2}}$. Then

$$
\begin{gathered}
\nabla \phi(x)=\frac{\nabla|\nabla u|^{2}}{(b-\varphi)^{2}}+\frac{2|\nabla u|^{2} \nabla \varphi}{(b-\varphi)^{3}} \\
\Delta \phi(x)=\frac{\Delta|\nabla u|^{2}}{(b-\varphi)^{2}}+\frac{4\left\langle\nabla \varphi, \nabla|\nabla u|^{2}\right\rangle}{(b-\varphi)^{3}}+\frac{2 \Delta \varphi|\nabla u|^{2}}{(b-\varphi)^{3}}+\frac{6|\nabla \varphi|^{2}|\nabla u|^{2}}{(b-\varphi)^{4}} \\
=\frac{2|\nabla d u|^{2}-\langle V, \nabla \varphi\rangle-2|\nabla u|^{4}}{(b-\varphi)^{2}}+\frac{4\left\langle\nabla \varphi, \nabla|\nabla u|^{2}\right\rangle}{(b-\varphi)^{3}} \\
+\frac{2 \cos \rho|\nabla u|^{4}-2\left\langle V, \nabla \varphi|\nabla u|^{2}\right\rangle}{(b-\varphi)^{3}}+\frac{6|\nabla \varphi|^{2}|\nabla u|^{2}}{(b-\varphi)^{4}}
\end{gathered}
$$

because

$$
\begin{gathered}
\langle V, \nabla \phi\rangle=\frac{\left\langle V, \nabla|\nabla u|^{2}\right\rangle}{(b-\varphi)^{2}}+\frac{2|\nabla u|^{2}\langle V, \nabla \varphi\rangle}{(b-\varphi)^{3}} \\
\frac{\langle\nabla \varphi, \nabla \phi\rangle}{b-\varphi}=\frac{\left\langle\nabla \varphi, \nabla|\nabla u|^{2}\right\rangle}{(b-\varphi)^{3}}+\frac{2|\nabla u|^{2}|\nabla \varphi|^{2}}{(b-\varphi)^{4}} \\
\frac{2|\nabla d u|^{2}}{(b-\varphi)^{2}}+\frac{2|\nabla \varphi|^{2}|\nabla u|^{2}}{(b-\varphi)^{4}} \geq \frac{4|\nabla \varphi||\nabla u||\nabla d u|}{(b-\varphi)^{3}} .
\end{gathered}
$$

Then (8) becomes

$$
\begin{aligned}
\Delta \phi(x) & \geq \frac{2 \cos \rho|\nabla u|^{4}}{(b-\varphi)^{3}}-\frac{2|\nabla u|^{4}}{(b-\varphi)^{2}}+\frac{2\langle\nabla \varphi, \nabla \phi\rangle}{b-\varphi}-\langle V, \nabla \phi\rangle \\
& \geq 2 \cos \rho(b-\varphi) \phi^{2}-2(b-\varphi)^{2} \phi^{2}+\frac{2\langle\nabla \varphi, \nabla \phi\rangle}{b-\varphi}+\langle V, \nabla \phi\rangle .
\end{aligned}
$$

Let $r^{2}=|F(x)|^{2}$ be the distance function in $\mathbf{R}^{n+1}$ from the point $x \in \boldsymbol{\Sigma}$ to the origin, $|\nabla r| \leq|\nabla F| \leq \sqrt{n}$. It is easy to check that $\Delta r^{2}=2(n-1)+2\langle\vec{H}, F\rangle$. Here and in the sequel, $\nabla$ and $\Delta$ are gradient and Laplacian operators on $\boldsymbol{\Sigma}$ with respect to induced metric respectively. Note that if the mean curvature of $\boldsymbol{\Sigma}$ is bounded, we have

$$
\Delta r^{2} \leq C(1+r),
$$

where $C$ depends only on $n$ and the upper bound of $|H|$.

Assume the origin is $0 \in F(\boldsymbol{\Sigma})$. If we denote $G=\left(R^{2}-r^{2}\right)^{2} \phi$, then there exists a point $x_{0} \in \boldsymbol{\Sigma} \cap \mathbf{B}_{R}^{n+1}(0)$ such that $\nabla G\left(x_{0}\right)=0, \Delta G\left(x_{0}\right) \leq 0$. Here $\mathbf{B}_{R}^{n+1}(0)$ is the ball with 0 as the center and $R(>r)$ as the radius in $\mathbf{R}^{n+1}$.

By a direct computation, we get

$$
\begin{gathered}
\nabla G=\left(R^{2}-r^{2}\right)^{2} \nabla \phi-4 r\left(R^{2}-r^{2}\right) \phi \nabla r=0, \\
\Delta G=\left(R^{2}-r^{2}\right)^{2} \Delta \phi-8 r\left(R^{2}-r^{2}\right) \nabla \phi \nabla r+8 r^{2} \phi|\nabla r|^{2}-2\left(R^{2}-r^{2}\right) \phi \Delta r^{2} \leq 0 .
\end{gathered}
$$


So,

$$
\frac{\nabla \phi}{\phi}=\frac{4 r \nabla r}{R^{2}-r^{2}}
$$

$$
\frac{\Delta \phi}{\phi}-\frac{2(C+1) r}{R^{2}-r^{2}}-\frac{C r^{2}}{\left(R^{2}-r^{2}\right)^{2}} \leq 0,
$$

where $C$ depends only on $n$ and $H$.

Then from (9), (10), and (11), we get

$$
\begin{aligned}
& 2 \cos \rho(b-\varphi) \phi-2(b-\varphi)^{2} \phi+\frac{2\left\langle\nabla \varphi, \frac{\nabla \phi}{\phi}\right\rangle}{b-\varphi}-\left\langle V, \frac{\nabla \phi}{\phi}\right\rangle \\
& -\frac{2(C+1) r}{R^{2}-r^{2}}-\frac{C r^{2}}{\left(R^{2}-r^{2}\right)^{2}} \\
& =\left[2 \cos \rho(b-\varphi)-2(b-\varphi)^{2}\right] \phi+\frac{\langle 4 r \nabla r, V\rangle}{R^{2}-r^{2}}+\frac{8 r\langle\nabla \varphi, \nabla r\rangle}{(b-\varphi)\left(R^{2}-r^{2}\right)} \\
& -\frac{2(C+1) r}{R^{2}-r^{2}}-\frac{C r^{2}}{\left(R^{2}-r^{2}\right)^{2}} \leq 0 .
\end{aligned}
$$

Because $T$ is a constant vector and $V$ is its tangent part on $\boldsymbol{\Sigma}$, the norm of $V$ is bounded. By this, the previous inequality becomes

$$
\begin{aligned}
& {\left[2 \cos \rho(b-\varphi)-2(b-\varphi)^{2}\right] \phi-\frac{C R}{R^{2}-r^{2}}-\frac{8 \sqrt{n} r|\nabla u|}{(b-\varphi)\left(R^{2}-r^{2}\right)}} \\
& -\frac{2(C+1) r}{R^{2}-r^{2}}-\frac{C r^{2}}{\left(R^{2}-r^{2}\right)^{2}} \leq 0 .
\end{aligned}
$$

By the definition of $G$, we have

$$
2(b-\varphi)(\cos \rho-(b-\varphi)) G-8 \sqrt{n} R G^{\frac{1}{2}}-C R^{3}-C R^{2} \leq 0 .
$$

By

$$
\cos \rho-(b-\varphi)=1-b>0, b-\varphi \geq b-\varphi(\Lambda)
$$

we get

$$
G-C R F^{\frac{1}{2}}-C R^{3}-C R^{2} \leq 0,
$$

where $C$ depends only on $n$, the upper bound of $|H|$, the norm of $V, b, \varphi$, and is independent of $R$.

Then,

$$
\sup _{\substack{B_{\frac{R}{2}}^{n+1}(0) \cap \Sigma\\}} G^{\frac{1}{2}}(x) \leq G^{\frac{1}{2}}\left(x_{0}\right) \leq C\left(R^{\frac{3}{2}}+R\right) .
$$

So,

$$
\sup _{B_{\frac{R}{2}}^{n+1}(0) \cap \Sigma} \frac{|\nabla u|(x)}{b-\varphi(u(x))} \leq C\left(\frac{1}{R^{\frac{1}{2}}}+\frac{1}{R}\right) .
$$

By taking $R$ to infinity, we know that the Gauss map must be constant, so Theorem 1.1 follows. 


\section{ACKnowledgements}

The authors are grateful to Professor Xiang Ma for his interest in this work and helpful conversations and for kindly pointing them to 8 . Both authors would like to thank the referee for numerous suggestions which helped to improve this presentation.

\section{REFERENCES}

[1] Hyeong In Choi, On the Liouville theorem for harmonic maps, Proc. Amer. Math. Soc. 85 (1982), no. 1, 91-94, DOI 10.2307/2043289. MR647905 (83j:53073)

[2] R. E. Greene and H. Wu, Function theory on manifolds which possess a pole, Lecture Notes in Mathematics, vol. 699, Springer, Berlin, 1979. MR521983(81a:53002)

[3] Jürgen Jost, Yuanlong Xin, and Ling Yang, The regularity of harmonic maps into spheres and applications to Bernstein problems, J. Differential Geom. 90 (2012), no. 1, 131-176. MR2891479

[4] Richard S. Hamilton, Harnack estimate for the mean curvature flow, J. Differential Geom. 41 (1995), no. 1, 215-226. MR1316556 (95m:53055)

[5] Gerhard Huisken and Carlo Sinestrari, Convexity estimates for mean curvature flow and singularities of mean convex surfaces, Acta Math. 183 (1999), no. 1, 45-70, DOI 10.1007/BF02392946. MR1719551 (2001c:53094)

[6] P. Li, Lecture notes on harmonic maps, Manuscript.

[7] Jiayu $\mathrm{Li}$ and Meng Wang, Liouville theorems for self-similar solutions of heat flows, J. Eur. Math. Soc. (JEMS) 11 (2009), no. 1, 207-221, DOI 10.4171/JEMS/147. MR 2471137 (2009m:58031)

[8] Jürgen Moser, On Harnack's theorem for elliptic differential equations, Comm. Pure Appl. Math. 14 (1961), 577-591. MR0159138 (28 \#2356)

[9] Yuanlong Xin, Mean curvature flow with convex Gauss image, Chin. Ann. Math. Ser. B 29 (2008), no. 2, 121-134, DOI 10.1007/s11401-007-0212-1. MR2392328 (2009b:53111)

[10] Y. L. Xin, Q. Ding, and L. Yang, The rigidity theorem of self shrinkers via Gauss maps, http://arxiv.org/abs/1203.1096.

[11] Mu-Tao Wang, Gauss maps of the mean curvature flow, Math. Res. Lett. 10 (2003), no. 2-3, 287-299. MR1981905 (2004m:53121)

Key Laboratory of Pure and Applied mathematics, School of Mathematics Science, Peking University, Beijing, 100871, People's Republic of China

E-mail address: chbao@126.com

Key Laboratory of Pure and Applied mathematics, School of Mathematics Science, Peking University, Beijing, 100871, People's Republic of China

E-mail address: ygshi@math.pku.edu.cn 